Preface

\title{
The Challenge of Treating Superbugs
}

\author{
David L. Paterson, MBBS, FRACP, FRCPA, PhD ${ }^{1}$ \\ ${ }^{1}$ University of Queensland Centre for Clinical Research, Brisbane, \\ Australia
}

Semin Respir Crit Care Med 2015;36:1-2.

Infections are a major cause of mortality in critically ill patients. It has been estimated that more than 100,000 health care-associated infections occur annually in intensive care units (ICUs) in the United States. ${ }^{1}$ This includes 50,000 cases of ventilator-associated pneumonia, 35,000 cases of catheterassociated urinary tract infections, and more than 15,000 cases of central catheter-associated primary bloodstream infection annually. ${ }^{1}$ In many ICUs, bacteria causing health care-associated infections are typically resistant to multiple antibiotics. In some situations, these bacteria are susceptible to three or fewer antibiotics (extensively drug resistant-XDR) and occasionally to all antibiotics (pan drug resistant-PDR). ${ }^{2}$ Thus, these might be truly termed as "superbugs."

In this issue of Seminars of Respiratory and Critical Care Medicine, we focus on the management of these superbugs.

Foremost in the minds of many as a superbug, is methicillin-resistant Staphylococcus aureus (MRSA). It is estimated that 10,000 cases of hospital-acquired MRSA bloodstream infections occur annually in the United States. ${ }^{1}$ In addition, there has been a dramatic increase in serious community-onset MRSA infections over the last decade. Vancomycin monotherapy has long been the standard of care for treatment of MRSA infections. In this issue of Seminars of Respiratory and Critical Care Medicine, there are reviews of emerging data on combination therapy for MRSA and also the multitude of new antibiotics now available to treat MRSA. Clearly, there is a significant change to the way in which we now view therapy of MRSA in critically ill patients, and these reviews are highly valuable in this regard.

Clostridium difficile associated diarrhea is now the most common health care-associated infection in the United States, with more than 80,000 cases estimated to occur annually. ${ }^{1}$ Many of these cases are due to a hypervirulent strain which is responsible for toxic megacolon and sometimes the need for colectomy. In elderly or compromised individuals $C$. difficile infection may be fatal, as a result of these complications. Since the advent of hypervirulent strains, there have been many advances in diagnosis and management of $C$. difficile infec-

Address for correspondence David L. Paterson, MBBS, FRACP, FRCPA, PhD, University of Queensland Centre for Clinical Research, Royal Brisbane and Women's Hospital Campus, Brisbane, Australia (e-mail: david.antibiotics@gmail.com). tions. Therefore, I have included a significant review on this infection in this issue of Seminars of Respiratory and Critical Care Medicine.

The multiresistant gram-negative bacilli truly fit the mold of superbugs, with many now being XDR or PDR. These include Pseudomonas aeruginosa, extended-spectrum $\beta$-lactamase producing Klebsiella pneumoniae and Escherichia coli, the carbapenem-resistant Enterobacteriaceae, Acinetobacter baumannii, Stenotrophomonas maltophilia, and other resistant nonfermentative gram-negative bacteria. Reviews on the management of each of these pathogens take their place in this issue, as well as a review on a major pathogen in tropical countries, Burkholderia pseudomallei (the cause of melioidosis).

Therapeutic strategies for these pathogens are covered in this issue of Seminars of Respiratory and Critical Care Medicine. Additionally, I have selected a review specifically on dosing colistin in critically ill patients. This state of the art review is particularly pertinent given the common situation where colistin is the only antibiotic to which many XDR gram-negative bacilli are susceptible. This work on colistin relies heavily on use of pharmacokinetic and pharmacodynamic (PK/PD) principles. The study of PK/ $\mathrm{PD}$ as it applies to optimizing dosing of antibiotics in the critically ill is further explored in another review in this series. Finally, a highly controversial area, the use of combination antibiotic therapy, is discussed in another timely article.

I would like to thank each of the authors who have contributed to this issue of Seminars of Respiratory and Critical Care Medicine. I am also very grateful for the Australasian Society for Infectious Diseases which hosted a highly successful "Gram Negative Superbugs" meeting in 2013 at the Gold Coast, Queensland, Australia. Presentations given at this meeting form the basis for many of the reviews in this issue. I trust that the information contained in these reviews will help optimize antibiotic therapy for the critically ill, and will contribute to saving lives around the world.
Copyright $\odot 2015$ by Thieme Medical Publishers, Inc., 333 Seventh Avenue, New York, NY 10001, USA.

Tel: +1(212) 584-4662.
DOI http://dx.doi.org/ 10.1055/s-0035-1544209. ISSN 1069-3424.
Superbugs; Guest Editor, David L. Paterson, MBBS, PhD, FRACP, FRCPA 


\section{References}

1 Magill SS, Edwards JR, Bamberg W, et al; Emerging Infections Program Healthcare-Associated Infections and Antimicrobial Use Prevalence Survey Team. Multistate point-prevalence survey of health careassociated infections. N Engl J Med 2014;370(13):1198-1208
2 Magiorakos AP, Srinivasan A, Carey RB, et al. Multidrug-resistant, extensively drug-resistant and pandrug-resistant bacteria: an international expert proposal for interim standard definitions for acquired resistance. Clin Microbiol Infect 2012;18(3): 268-281 